\title{
LIGHT ADAPTATION AT THE FOVEA FOR NORMAL EYES
}

BY

\author{
W. D. Wright, D.SC., A.R.C.S. \\ IMPERIAL COLLEGE OF SCIENCE AND TECHNOLOGY
}

\section{Aim of the Investigation}

As a result of experiments on the foveal adaptation process by the method of binocular matching, 2 , it seemed likely that data obtained from observers with defective vision might lead to further knowledge of the retinal processes and of certain diseases of the eye and might in some cases assist in their diagnosis. Before this possibility could be tested, it was essential to determine the differences that might exist among apparently normal eyes, for without this information the significance of variations from the normal could not be assessed. The results given below are therefore, in a sense, preliminary observations, but they have proved to be of considerable interest in themselves, apart from their value in connection with any subsequent investigation.

The earlier work quoted above was carried out using an elaborate trichromatic colorimeter with which both intensity and colour changes induced by various adapting conditions, were recorded. To avoid prolonging the investigation unduly, the number of observers had to be strictly limited and in practice nearly all the observations were made by the author. In order that a much greater number of observers might be tested, it was necessary to restrict the scope of the observations and to simplify them as far as possible, both to save time and to ensure that those not familiar with visual observations would have the minimum of difficulty in carrying out the tests. For this purpose a subjective photometer ${ }^{3}$ was constructed with which tests could be carried out using a single adapting radiation and recording intensity changes alone. With this instrument, over 100 observers have been tested and it is with the analysis of these results that this paper is concerned.

\section{Apparatus and Technique}

A first model of the subjective photometer has been described elsewhere. ${ }^{3}$ This instrument was used for the initial observations, but an improved apparatus was designed and used in the majority of the tests reported below. The later instrument is illustrated in Figs. 1 and 2. 
A calibrated " fullolite" lamp L is mounted in the lamphouse $\mathrm{H}$ and used to illuminate two optical systems via the aperture $A_{1}$ for the right eye system and the aperture $A_{2}$ and the mirror $M$ for the left eye system. Each system consists essentially of a

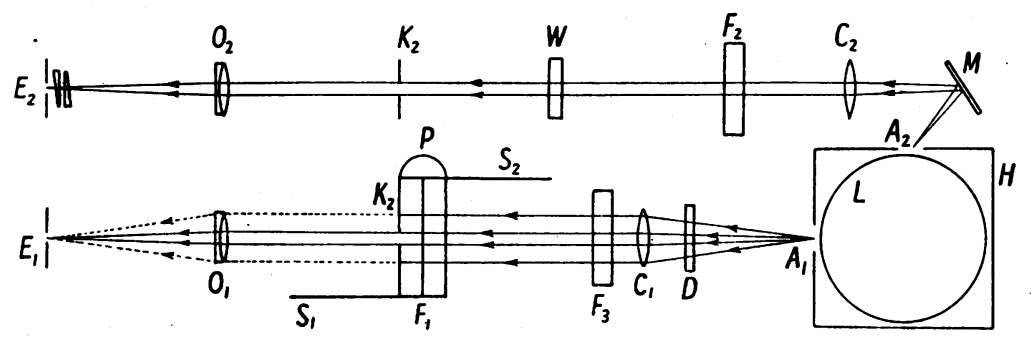

FIG. 1.

Optical system of subjective photometer.

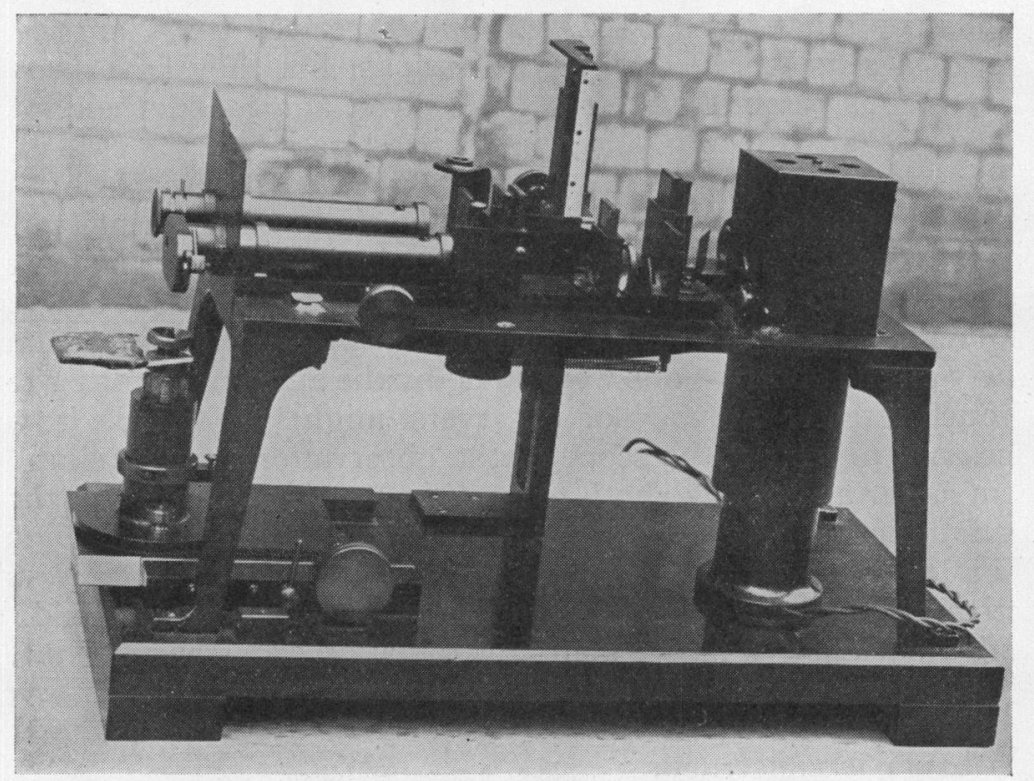

FIG. 2.

General view of subjective photometer.

collimating lens, $\mathrm{C}_{1}, \mathrm{C}_{2}$, and an observing lens, $\mathrm{O}_{1}, \mathrm{O}_{2}$. Light from the surface of the lamp is rendered parallel by $C_{1}$ and $C_{2}$ and brought to a focus by $\mathrm{O}_{1}$ and $\mathrm{O}_{2}$ at the two exit-pupils, $E_{1}$ and $E_{.2}$ These consist of holes drilled in the end plates of the observing tubes. Filter holders are provided in each beam for 
suitable neutral filters and colour filters. A calibrated photometric wedge, $\mathrm{W}$, is mounted in the left eye beam to enable the observer to vary the intensity of that beam by known amounts.

The test fields are defined by two horizontal rectangular apertures, $\mathrm{K}_{1}, \mathrm{~K}_{2}$, of a size such that they subtend an angle of approximately $1^{\circ} \times 2^{\circ}$ at the eye. Their colour and intensity are controlled by filters at $F_{1}$ and $F_{2}$. When making binocular matches it is desirable that the test patches should only be viewed momentarily, otherwise the eye tends to become to some extent adapted to them, and their apparent brightness will be correspondingly modified. As a general rule, it is found to be satisfactory if the patches are viewed for one or two seconds in every

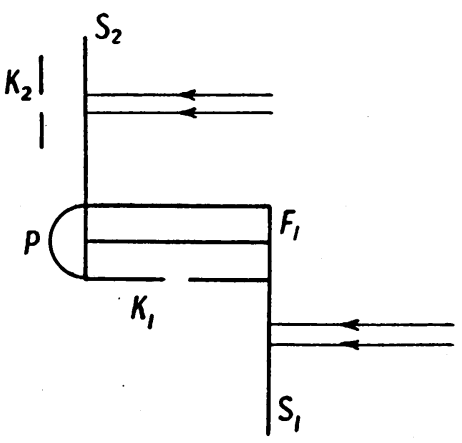

(1)

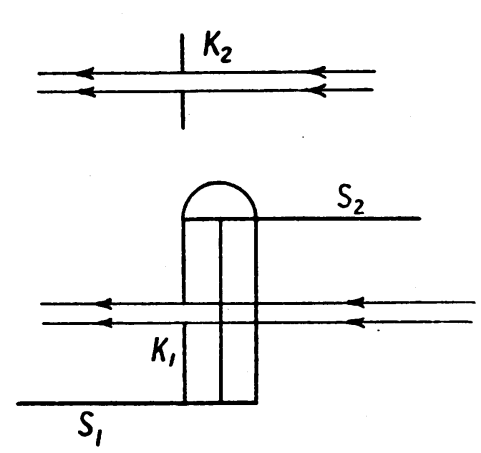

(2)

FIG. 3.

Operation of shutters on photometer.

ten seconds and this is allowed for, by mounting the aperture $K_{1}$ and the filters at $F_{1}$ on a pivot $P$. This pivot can be operated by a knurled head underneath the main platform on which the apparatus is mounted and can be rotated by hand through a right angle. Attached to $F_{1}$ are two shutters, $S_{1}$ and $S_{2}$, and when $P$ is rotated, $K_{1}$ and $F_{1}$ move out of the field of view and simultaneously $S_{1}$ and $S_{2}$ move in front of the right and left beams respectively to leave an unilluminated field in each eye. The two positions of $\mathrm{P}$ are illustrated diagrammatically in Fig. 3. A spring beneath the table maintains the shutters normally in position 1 of Fig. 3, so that the test fields only become visible when $P$ is rotated to position 2 . When necessary the mechanism can be fastened by a catch in the latter position, to leave the test patches permanently in the field of view.

The adapting field is introduced by raising the shutter $S_{1}$ when in position 1. This allows the whole of the light from $\mathrm{C}_{1}$ to 
enter $\mathrm{O}_{1}$, so that the right eye views a circular field about $6^{\circ}$ angular subtense, the lett eye remaining dark. The colour and intensity of the adapting field can be controlled by a filter at $\dot{\mathrm{F}}_{3}$ and a daylight filter at $\mathrm{D}$. In the experiments to be described a white adapting field was employed and this, of course, was independent of the filters at $F_{1}$. On the other hand, the right eye test patch was controlled by both the filters at $F_{1}$ and at $F_{3}$, and the filters at $F_{1}$ had to be selected after the action of those at $F_{3}$ had been allowed for.

The distance between the exit-pupils, $E_{1}$ and $E_{2}$, has to be adjustable to suit the inter-ocular distance of each individual. This is provided for by mounting the two optical systems on rectangular bars pivoted at one end. Adjusting screws and springs allow each bar to be moved independently and when correctly adjusted, they can be clamped in position. Owing to the Stiles and Crawford effect, it is important that the exit-pupils should be accurately centred relative to the observer's pupils. This calls for accurate adjustment of the pupils and, equally important, rigid location of the observer's head. The wax dental impression on which the observer bites to fix his head, was again used, but was attached to an improved mounting, shown in Fig. 2 , which provided adjustments for squaring-on the observer's head, bringing his eyes to the correct distance from the instrument and also to the correct height.

The left eye is centred first, the vertical adjustment on the mouth-piece and the horizontal adjustment on the left eye optical system being used to bring the instrument pupil and eye pupil on to the same axis. This adjustment is controlled by viewing a coarse grating placed in the field of view and illuminating it by light which has passed through a purple cobalt filter. When the pencil of light emerging from the exit-pupil does not pass centrally through the cornea, some unsymmetrical chromatic aberration is introduced by the eye, which causes the bars of the grating to be illuminated on one side by red light and on the other by blue. The effect is, of course, apparent when white light is used, but is more marked and the adjustment is more sensitive, with the cobalt glass. A vertical grid is used to test for horizontal alignment, a horizontal grid for vertical alignment. It is estimated that the adjustment can be repeated at least to within $0.25 \mathrm{~mm}$.

Having centred the left eye, the position of the mouthpiece mounting can no longer be altered to centre the right eye. This adjustment has to be made solely by moving $E_{1}$, and the chief movement required is a horizontal one to give the correct interocular separation. Provided the observer's eyes and mouth are reasonably symmetrical, the vertical adjustment required is not 
usually more than 2 or $3 \mathrm{~mm}$., and this displacement of $E_{1}$ is sufficiently small to be provided by a simple vertical movement of $E_{1}$ off the axis of the system, without risk of producing any vignetting or change in intensity of the field. In one or two cases when a bad dental impression had been made, it has been necessary to make a second impression, in order that the right eye could be centred within the limits of motion provided in the apparatus.

The remaining adjustment is one to bring the two test patches apparently vertically above one another when seen simultaneously. A small vertical separation is also convenient to prevent binocular rivalry and to remove all chance of fusion of the two patches. As the relative positions of the patches is governed by the state of convergence of the two eyes; the adjustment must be made with the eyes as nearly at rest as possible. The actual control of the position of the patches is obtained through two prisms mounted just in front of $E_{2}$, each prism being capable of independent rotation.

At first sight these adjustments appear formidable, but an observer familiar with what is required can complete the settings in 3 or 4 minutes. For new observers, some fifteen minutes may be needed and rather more if the making of the dental impression is included.

Having made the adjustments, the observer is then allowed to dark adapt for 20 minutes, curtains being used to prevent light from the apparatus reaching his eyes. At the end of this period, the first observation is recorded, namely the setting of the photometer wedge when both patches appear of the same intensity. Three settings are made and, as mentioned above, the patches are only viewed for 1 or 2 seconds in every 10 . The right eye is now light adapted for 3 minutes and at the end of this time, the test patches are viewed periodically for 1 second in every 10 , and the photometer wedge is adjusted until the test patches again appear equally bright. Three readings are again recorded. Finally, the adapting field is removed and the test patches are brought permanently into the field of view. A series of rapid matches are then made to record the recovery of sensitivity of the right eye, the time of each reading being noted with a stop watch. When this observation has been made, the observer is given 10 minutes' rest, after which the whole of the observations from the commencement of the light adaptation, are repeated. A complete sitting, including adjustments and dark adaptation, occupies about $1 \frac{1}{2}$ to 2 hours.

In nearly all the cases reported here, the observer had two sittings on different days, the occasions sometimes being separated by several weeks. In a few cases, when the agreement between 
the two sets of results was unsatisfactory, a third sitting was held.

The white adapting radiation that was used had a colour temperature of about $4,400^{\circ} \mathrm{K}$ and gave a retinal illumination of 6,000 photons. The test patches were red, as given by Ilford's red spectral filters, and had a retinal intensity of about 30 photons.

In presenting and discussing the results the following symbols have been used :

A $\quad \ldots$ adaptation intensity.

a $\quad$... apparent brightness of test patch when both eyes dark adapted.

$a_{0} \quad \ldots$ apparent brightness of test patch immediately after light adapting the right eye.

$a_{\mathrm{t}} \quad \ldots$ apparent brightness of test patch at time $t$, in seconds, from the end of the light adaptation.

$p \quad$.. the intra-ocular factor, that is, the sensitivity of the right eye compared to the left with both eyes dark adapted.

$q=a / a_{0}$ the adaptation factor, that is, the ratio between the apparent brightness of the test patch before and after light adaptation.

In all the observations the value of $a$ was arbitrarily adjusted to be equal to 1,000 . To do this the instrument reading obtained from the photometer wedge calibration, had to be multiplied by a factor which depended on the relative sensitivities of the two eyes. With both eyes equally sensitive, the scale was arranged for convenience to give a reading of 1,000 directly. If the right eye were twice as sensitive as the left $(p=2)$, then twice as much light would be required in the left as in the right eye to give a photometric match, that is to say, the instrument reading would be 2,000 . Hence $p$ is derived by dividing the instrument reading by 1,000 . The values of $a_{0}$ and $a_{t}$ were similarly adjusted to refer to a test patch having an initial intensity of 1,000 , that is, the instrument readings were multiplied by $1 / p$.

The reading at which two equally sensitive eyes would make a match was found by matching (binocularly) each beam in turn against a third reference patch.

\section{Validity and Significance of the Observations}

The three most important pieces of information to be obtained from the observations are (1) the relative sensitivity $p$, of the two eyes when in the same state of adaptation; (2) the change in sensitivity produced by a given state of adaptation, i.e., $q$; and (3) 
the shape of the recovery curve showing the course of recovery back to the initial sensitivity.

The evaluation of the relative sensitivity of the two eyes from the magnitude of $p$ is obviously straightforward, but the accuracy to which $p$ can be determined is not high. Variations of 20 or 30 per cent. are quite common when repeating observations from day to day, even by persons familiar with the apparatus. These variations may arise from actual changes in the sensitivity of the eyes, from errors in adjustment or from changes in the apparatus, but the last mentioned could contribute only a small part of such a large uncertainty. Values of $p$ which differ from unity only by small amounts are therefore of little significance, but when $p$ is greater than 2 or less than $\frac{1}{2}$, some physiological abnormality may be indicated. Occasionally cases may arise where for some reason the response in one eye tends to inhibit that in the other, in which case only one patch can be seen at a time. If the light to the dominant eye is then obstructed, the patch in the other eye immediately becomes visible, so that a comparison of the sensitivities of the two eyes may have to be made by viewing each patch alternately and matching by memory.

While the reality and significance of $p$ is hardly in doubt, the interpretation to be placed on $q$ is a matter for some speculation. The underlying justification of the binocular matching method is that the left eye is not materially affected by the light adaptation in the right eye and can accordingly be used to provide a reference scale. It is unlikely that this condition is strictly satisfied, but it is possible to make one or two tests which demonstrate that whatever changes may be induced in the left eye, they are relatively unimportant. Thus if, while viewing a test patch in the left eye, a bright light is projected into the right eye, the brightness of the test patch shows no appreciable change. Neither is any significant change produced when the bright light is removed. The accuracy of a comparison made in this way is not high, but a change in sensitivity of 20 per cent. should be easily visible. Again, if a yellow test patch is viewed in the left eye and a red adapting light is thrown into the right eye, no noticeable colour change is induced in the test patch, in spite of the fact that the eye is very sensitive to any changes in hue in a yellow test colour. When very faint test patches are used some small changes do become apparent, which would suggest that something in the nature of a faint after-image is formed in the unadapted eye. It is, however, of little practical importance owing to its small magnitude.

One objection to recording $q$ with the subjective photometer instead of a colorimeter is that, although the colour of the test patch in the left eye is effectively unchanged by the adaptation 
in the right eye, the same does not strictly hold for the right eye test patch, even with the white adaptation. The colour change is not large, but it is easily seen that the two patches are not quite the same colour, at least in the early stages of the recovery curve. This leads to a further lowering of the accuracy of the observations which, in view of the inherent difficulties in binocular matching, is obviously not comparable to that obtained in normal photometer. Hence binocular matching with the subjective photometer can only be applied to measurements of gross effects, but that proves to be hardly any limitation at all, since the changes that do occur are usually very large.

Some doubts have been expressed as to the time at which $q$ is actually recorded. In the apparatus that has been used in these experiments, an appreciable fraction of a second elapses from the time the adapting light is removed to the time the test patches become visible. It is probably true to say that the match is not made until $\frac{1}{2} \mathrm{sec}$. or $1 \mathrm{sec}$. has passed after the adapting light is removed. There is ample evidence $(4,5,6)$, that rapid " off " effects occur when a stimulus is removed, and in the present experiments they will all be missed. These rapid reactions are apparently electrical in origin and are complete in so short a time that there is little reason to suppose that they play any part in the recovery process as we have measured it. It is, therefore, reasonably safe to assume that the factor $q$ and the recovery curve record only those processes that are photo-chemical in origin.

\section{Results}

In all, 118 observers have been tested to date (June, 1938), 17 on the first model of the subjective photometer, 101 on the new model. The former served to demonstrate the value of further investigations, and as the tests were not made under such well controlled conditions as those made with the new apparatus, the results have not been included except.when analysing the percentage distribution of different types of recovery curve. Eleven of the observers on the new instrument were known to be suffering from some visual disease and, with one exception, their results have also been omitted from the present report.

As indicated above, the observations are by no means easy and it was found that certain of the results, generally the recovery curves, were for some observers so poor that no use could be made of them. Accordingly some 10 per cent. of the curves have been discarded. About 25 per cent. of the curves were good, the agreement of the two observations on the same day being satisfactory and the mean curves for the two days also agreeing well. 
The remainder of the observers gave curves in fair agreement and would no doubt have been better, if it had been possible to spend time in practice observations.

The Intra-ocular Factor, p.-The sensitivity of the right eye relative to the left eye is shown for 101 observers on a logarithmic scale in Fig. 4. The number of observers has been plotted who recorded a value of $p$ within each $0 \cdot 1$ of the $\log _{10} p$ abscissa, and

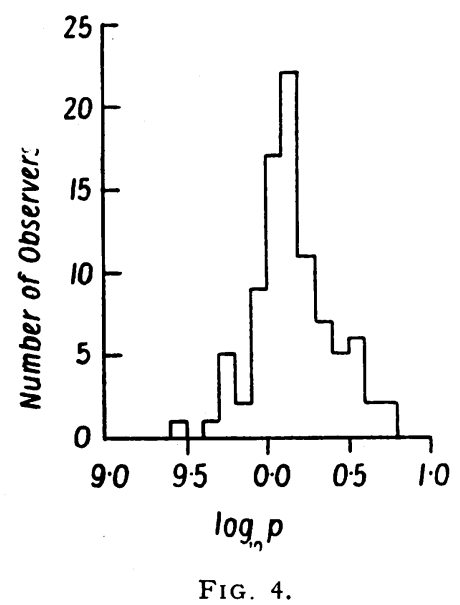

Distribution of measurements of intra-ocular factor, $p$. Number of observers occurring in each 0.1 of abscissa is plotted.

it is seen that the distribution has the general shape of a probability curve. The maximum occurs slightly to one side of the zero value, and while this might indicate a slight " right-eye" dominance of the observers, it is more probably due to an error in calculating the intra-ocular intensities, a binocular observation not easy to carry out with certainty.

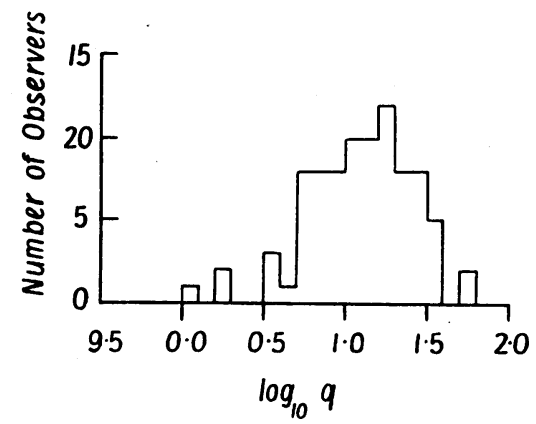

FIG. 5 .

Distribution of measurements of adaptation factor, q. Number of observers occurring in each $0 \cdot 1$ of abscissa is plotted. 
Taking the maximum of the curve as representing the true equality of sensitivity of the two eyes, it appears that for 20 of the observers one eye is more than twice as sensitive as the other and for two or three observers, one eye is more than four times as sensitive as the other.

The Adaptation Factor, $q$.-The values of $q$ for 85 observers are shown in Fig. 5, in which the number of observers who

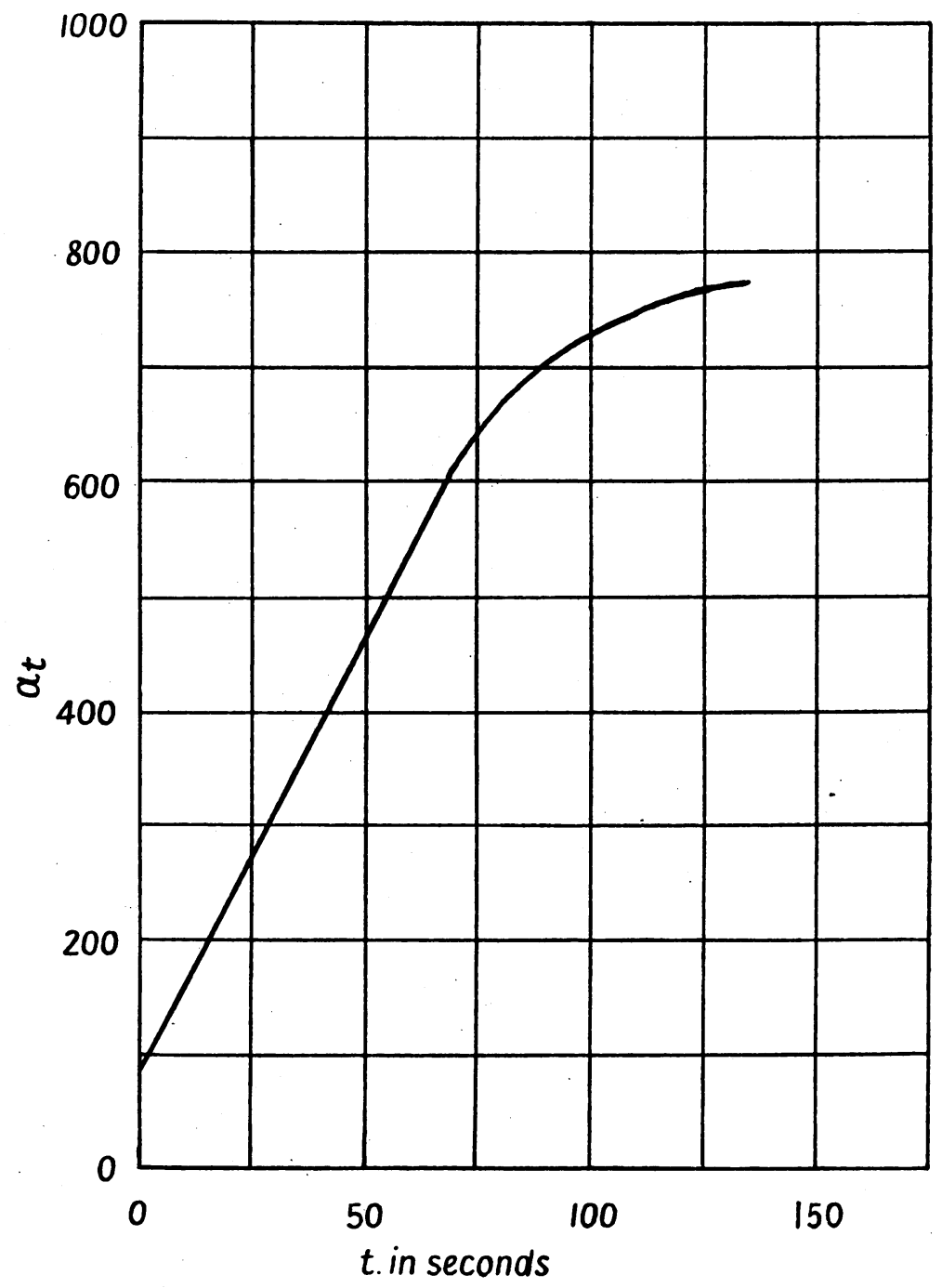

FIG. 6.

Recovery curve of direct type. Mean of 36 observers. 
Light Adaptation at the Fovea for Normal Eyes

recorded a value of $q$ in each 0.1 of the $\log _{10} q$ abscissa, is plotted. A very wide spread of the observations is apparent, with no well defined maximum occurring in the central block of the results. So far as any mean value of $q$ has any significance, it may be taken as about $q=12.5\left(\log _{10} q=1 \cdot 1\right)$. That, of course, holds only for the given adaptation intensity of 6,000 photons. For a

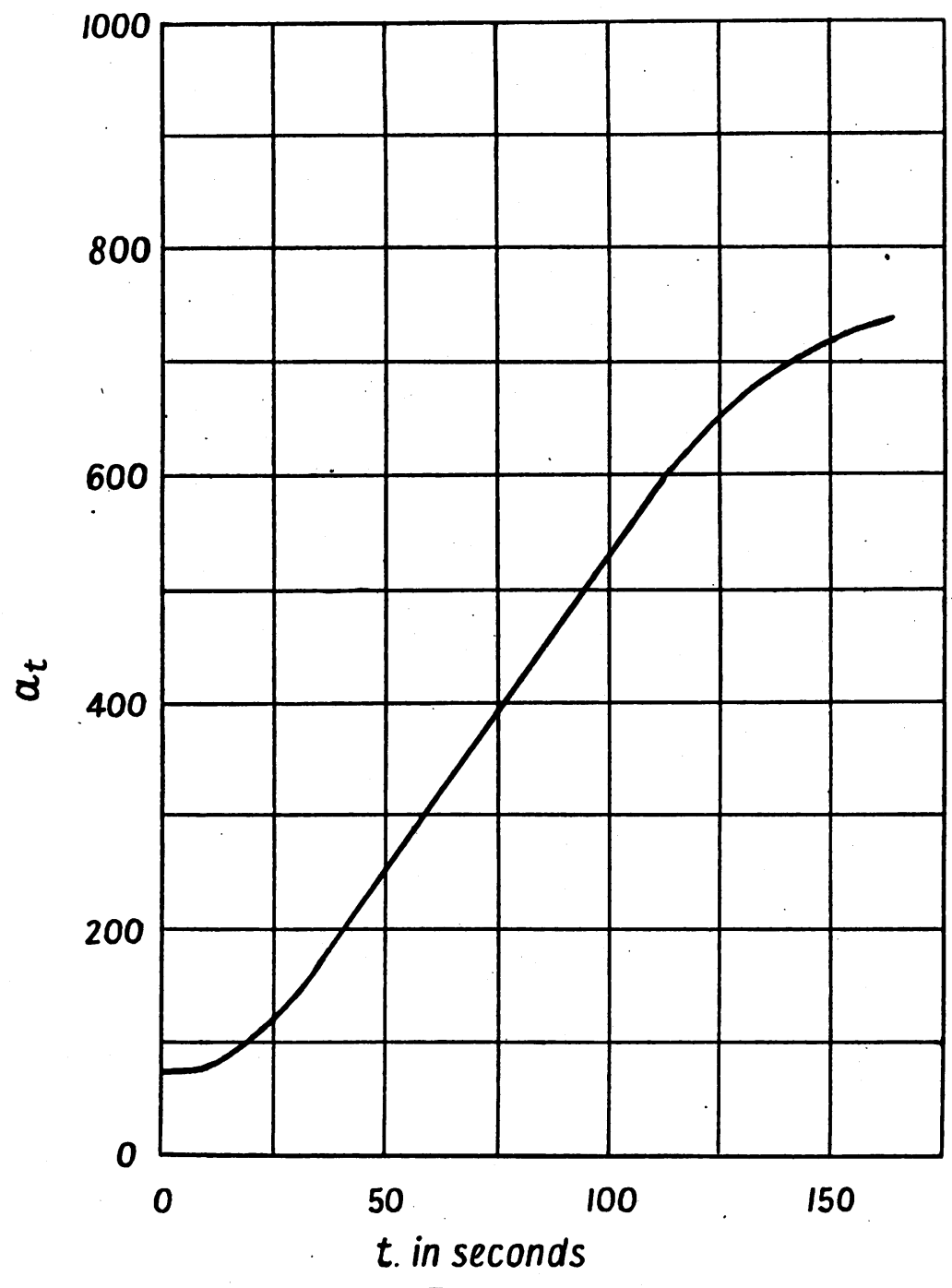

Fig. 7.

Recovery curve of delayed type. Mean of 26 observers. 
few of the observers, it will be seen that $\log q$ is exceptionally small, an abnormality which is discussed later.

The Recovery Curve.- It has been found that there are two general types of recovery curve, those which show a recovery process that sets in as soon as the adaptation is removed (direct recovery), and those in which for some few seconds no recovery is apparent and in which some further small loss in sensitivity may even occur (delayed recovery). The two types of curve are illustrated in Figs. 6 and 7, which represent the mean of 36 direct recovery curves and 26 delayed curves respectively. There is a very well marked difference between these two diagrams and there is no doubt whatever that observers exist with a difference in recovery process as definite as that shown here. On the other hand, these curves are mean curves derived from a number of observations which show a considerable spread among themselves and the two groups each contain borderline cases for which classification is difficult. This spread is illustrated in Fig. 8, where the 36 values of $a_{50}$ for the direct recovery cases have been plotted on a logarithmic scale in Fig. $8 a$, and the 26 values of $a_{50}$ for the delayed curves are shown in Fig. $8 b$. An appreciable amount of overlapping occurs, although it does not follow that observers with values of $a_{50}$ which occur in the overlap, are necessarily intermediate cases between direct and delayed recovery, since this will also be governed by the associated value of $a_{0}$. Out of 92 observers, 46 (50 per cent.) had direct curves, 32 (35 per cent.) had delayed curves, and 14 (15 per cent.) were intermediate or too indefinite to classify.
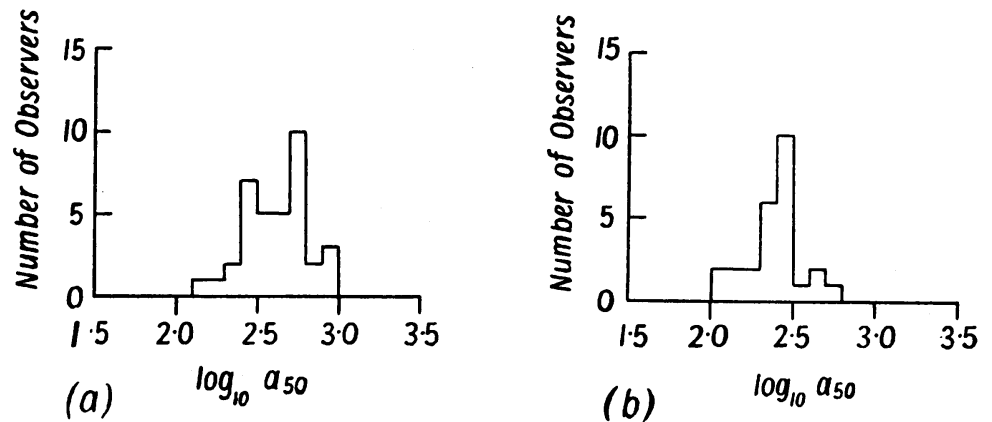

FIG. 8 .

Spread of observations of recovery curve 50 seconds after removal of adapting light. (a) $\log a_{50}$ for direct type recovery curve. Number of observers occurring in each 0.1 of abscissa is plotted. (b) $\log a_{50}$ for delayed type recovery curve. Number of observers occurring in each $0 \cdot 1$ of abscissa is plotted. 
Abnormal Recovery Curves.-Two abnormal results may be mentioned. In the first, it was found that those whose sensitivity was reduced to a relatively small extent by the light adaptation ( $q$ small) showed a rapid recovery curve. A typical case is illustrated in Fig. 9. It was, moreover, usually the case that these

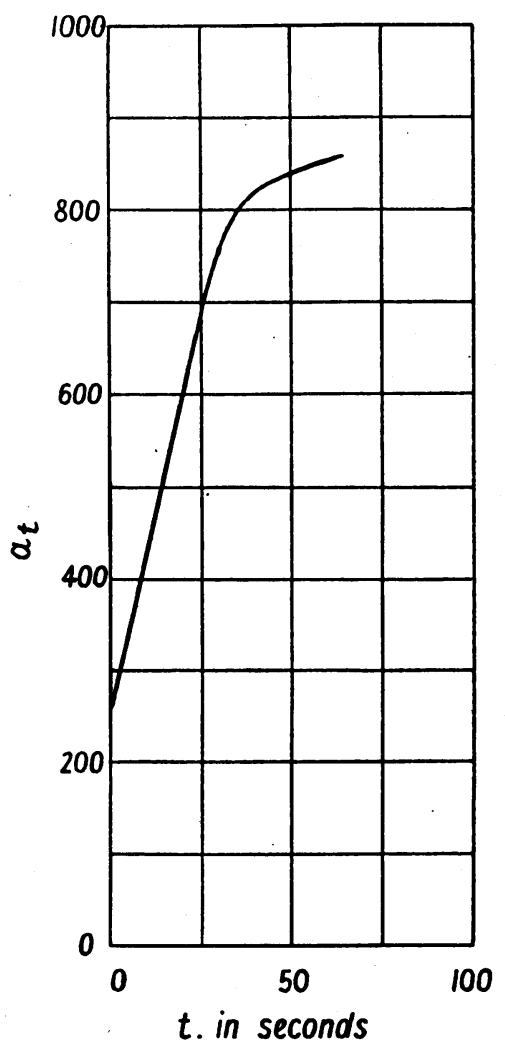

FIG. 9.

Recovery curve for observer having small adaptation factor.

observers were conscious of being unusually sensitive to glare ; the same was also true of one or two observers for whom $q$ was approximately 1 (no loss of sensitivity). The value of $q$ was in these cases generally rather indefinite, but that such observers should exist, is very remarkable. The correlation between rapid recovery and susceptibility to glare needs further observations for confirmation; but it is of considerable interest and is discussed later.

The other abnormal case was that of a monochromat. This observer, a girl, had already been tested on a colorimeter to 
confirm the monochromasy and to measure her luminosity curve. She also showed the other signs usually accompanying the defect, namely poor visual acuity and slight nystagmus. The adjustments of the subjective photometer presented some difficulties, but they were not insuperabie. The greatest problem lay in actually obtaining the observations, since the observer was unable to see both patches simultaneously. A shutter was accordingly constructed and worked by the person operating the apparatus in

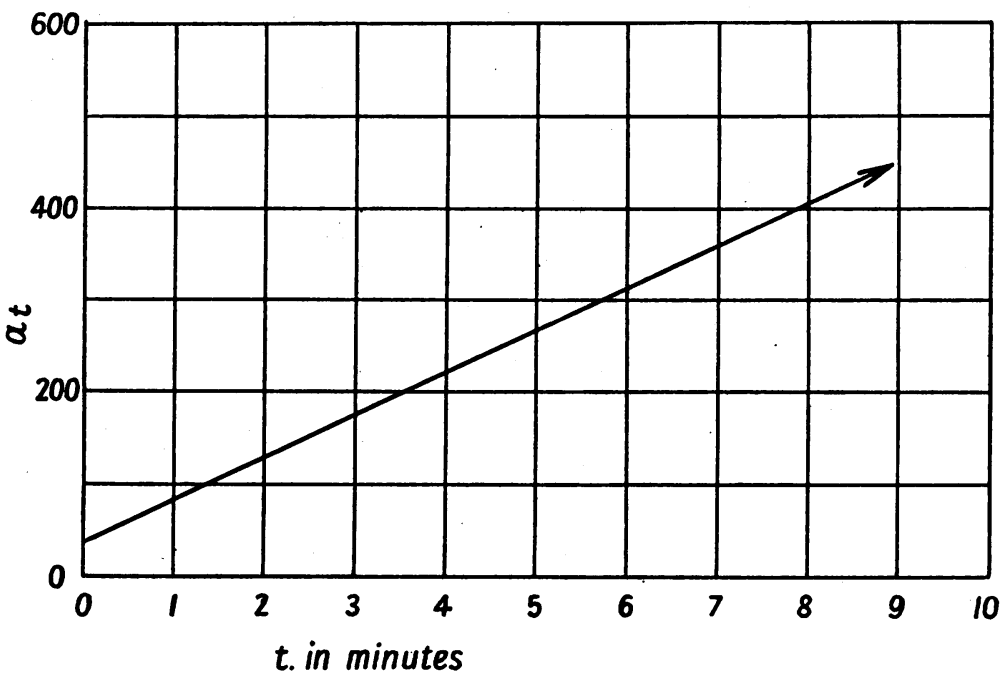

FIG. 10.

Recovery curve for monochromat.

such a manner that each patch was revealed in turn and the match was made by memory. In this way, a complete set of observations was possible, including the recovery curve, but the recording of the latter was only possible because it happened that the recovery was very slow and no appreciable change in sensitivity took place while the memory match was being made. The mean curve for three sets of observations taken on two different occasions is shown in Fig. 10. It is to be noted that the time scale is in minutes, so that the recovery is very much slower than the normal.

\section{Discussion}

The Intra-ocular Factor.-It is not, apparently, very abnormal for an observer to have one eye twice as sensitive as the other. A ratio of 4, on the other hand, is abnormally high and further examination of the person's vision would seem to be indicated. 
Apart from its significance as suggestive of some more deep-seated trouble, a big difference in light sensitivity may in itself be responsible for other signs of visual defect, such as a reduced visual acuity due to the lower subjective brightness of the test chart, a discrepancy that might disappear if the illumination on the chart were raised for the eye concerned. Again, with one eye contributing to the brain only a fraction of the total visual response, a reduced stereoscopic acuity might also be expected.

Adaptation Factor and Recovery Curve.-The results for the adaptation factor show that even among apparently normal persons there is a wide variation in the extent to which light adaptation depresses the sensitivity. If pathological conditions are found to affect this factor, the changes produced will have to be very considerable for their existence to be demonstrated with certainty or to be of any practical use. This aspect can only be tested by further experiments.

Assuming that the correlation between a small value of $q$, rapid recovery and susceptibility to glare, is confirmed, the condition must presumably be due to the presence in the retina of an active photochemical substance having a high rate of regeneration. A balance between decomposition and regeneration would then occur at a high photochemical concentration, that would lead to an abnormally large visual response. Such an observer would therefore be peculiarly sensitive to light.

For those observers who adapt to a greater extent (large $q$ ) and have a direct, but slower, recovery curve, there should be less discomfort from high intensities, at least once the observer has become accustomed to the light. The balance between decomposition and regeneration would then occur only after the photochemical concentration had been considerably reduced, and the visual response would not be abnormally large. When first exposed to a high intensity, the concentration might be sufficiently high to cause a strong sense of glare, but this should soon disappear.

It is difficult to deduce anything about the photo-chemical condition of the observers with delayed recovery curves. From considerations of a balance point, the light sensitive substance should be almost completely decomposed before any balance would be reached with a process that apparently does not begin to recover for some seconds after the light source is removed. In that case a very high value of $q$ would be expected, but in practice this is not found. It may be that the regeneration process is different when the light is acting from when the stimulus is removed or alternatively the total amount of light sensitive material in addition to its concentration may be the distinguishing feature. At the moment this can only be a matter for speculation. 
No doubt variations in the metabolism of the retina from individual to individual will eventually have to be taken into account. Two instances may be quoted to illustrate this possibility : deficiency of vitamin $\mathrm{A}$, which is known to affect visual purple regeneration (see e.g., ${ }^{7},{ }^{8}$ ), and the effect of glucose on visual purple regeneration, as demonstrated by Therman. ${ }^{9}$

It would obviously be advantageous if the work on foveal adaptation could be linked up with measurements of the dark adaptation process. There is a good deal of activity at present in this field, especially in relation to pathological conditions of the eye, ${ }^{7}$ and the results are complementary to those of the present paper. It would, for example, be very interesting to determine whether abnormalities in the rod mechanism are accompanied by similar abnormalities in the cone mechanism.

\section{Acknowledgements}

I should like to express my gratitude to the Medical Research Council for the special grant they provided for building the subjective photometer and to cover the expenses of the work, and also to the authorities at the Royal London Ophthalmic Hospital, Moorfields, for their help in providing facilities and accommodation to undertake the work. I have also to acknowledge my thanks to Mr. J. H. Nelson for assisting in the design of the apparatus and to Miss W. M. McKeon, who was responsible for recording the results of many of the observers. Lastly, I have to thank the observers themselves for assisting in such an essential manner in the investigation.

\section{Summary}

A new model of the subjective photometer is described, in which the effects of adapting the eye to a given intensity, are recorded by matching the test patch viewed in the light adapted right eye against a similar patch seen in the constantly dark adapted left eye. Observations on more than 100 observers have been recorded and are analysed. The data include the intra-ocular factor, that is, the sensitivity of the right eye relative to the left when both eyes are dark-adapted, the adaptation factor, which gives the factor by which the sensitivity of the eye is reduced for the given adaptation intensity (6,000 photons), and the recovery curve, which records the recovery of sensitivity after the adapting light has been removed. Among normal observers, two types of recovery curve have been found, a direct and a delayed recovery, in the latter of which no recovery is recorded for the first few seconds after the removal of the adapting light. The results are discussed in relation to the susceptibility of different observers to glare, to 
the photo-chemical reaction in the retina and to the application of the method for pathological investigations.

\section{REFERENCES}

1. W. D. Wright.-Proc. Roy. Soc., B, Vol. CXV, p. 49, 1934.

2. Proc. Roy. Soc., B, Vol. CXXII, p. 220, 1937.

3. and J. H. Nelson.-Jl. Sci. Inst., Vol. XII, p. 373, 1935.

4. R. Granit and P. O. Therman.-Jl. Physiol., Vol. LXXXIII, p. 359, 1935.

5. H. K. Hartline.-Amer Jl. Physiol., Vol. CXXI, p. $400,1938$.

6. J. F. Schouten.-Visueele meting van Adaptatie. Utrecht, 1937.

7. J. B. FELDMAN.-Arch. of Ophthal., Vol. XIX, p. 882, 1938.

8. G. Wald, H. Jeghers and J Arminio.-Amer. Jl. Physiol., Vol. CXXIII, p. $732,1938$.

9. P. O. Therman.-Acta Soc. Sci. Fenn, Helsingfors, Ser. B, 2, No. 1, 1938.

\section{ANNOTATIONS}

\section{Sulphanilamide in the Treatment of Trachoma}

In the Lancet of October 29, 1938, appeared an article by three members of the Sudan Medical Service on this subject. Kirk, McKelvie and Hussein have tried prontosil album, given in tablet form by the mouth, in weekly courses with a week's interval between each course. The dose of each tablet, $7 \frac{1}{2}$ gr. thrice daily (proportionately reduced for children), was selected arbitrarily.

The paper is based on the results in 25 cases of adults. A convenient table allows the main facts to be seen at a glance. The results appear to be encouraging, especially as regards the corneal complications of pannus and keratitis. The treatment is simple and less painful than the local treatment of the disease, but it is, of course, too early to say whether the results will be permanent. It may be noted that cases of trachoma complicated by spring catarrh did not do so well as uncomplicated cases.

Ophthalmologists throughout the world will be anxious for further first-hand reports on the use of this drug in cases of trachoma.

\section{Volume XXIII}

With the completion of our 22 nd volume this Journal may fairly be said to have passed out of the stage of adolescence. Recently the amount of original material in hand has been greater than usual and the Editorial Committee has considered the question of increasing the size of the monthly numbers. When the project of a British Journal of Ophthalmology was first being discussed in 1916 the hope was expressed that it would be possible to issue a 64 page number every month and even a larger number of pages if thought 